\title{
Sind Supervision und Coaching in unendlich vielen Feldern nutzbar?
}

\author{
Astrid Schreyögg
}

Angesichts des vorliegenden Heftes lässt sich die Frage, ob Supervision und Coaching in unendlich vielen beruflichen Feldern zu nutzen sind, eigentlich nur mit „Ja“ beantworten. Supervision, die aus der amerikanischen Sozialarbeit hervorging, wird zwar bis heute überwiegend in Milieus der Human Services praktiziert. Sie hat sich dabei aber weit über die klassische Sozialarbeit hinaus in klinische, schulische und sonstige soziale Dienstleistungsbereiche vorgearbeitet. Und Coaching, das ursprünglich der US-amerikanischen Personalwirtschaft entstammte, dehnt sich bis heute ebenfalls weit aus auf verschiedene Organisationen der öffentlichen Verwaltung, auf den Hochschulbereich, aber auch auf soziale Dienste. Im Zuge dieser Entwicklungen dienten beide Formate auch in interkulturellen Kontexten zur Verständigung und zur verbesserten Integration. Mit dem vorliegenden Heft möchten wir die Leser etwas teilhaben lassen an dieser Vielfalt der Felder, in die Supervision und Coaching bis heute vorgestoßen sind.

Im ersten Beitrag berichtet Peter Breu von der Bedeutung, die Coaching heute in manchen Organisationen der öffentlichen Verwaltung für das betriebliche Gesundheitswesen hat. Der Autor betont, dass durch die Entwicklung der letzten Jahre zum New Public Management ein enormes Konfliktpotenzial in diesen Milieus aufgebrochen ist. Dieses geht mit hohen psychophysischen Belastungen einher. Coaching kann dann einen wichtigen Beitrag zur Reduktion dieser Belastungen leisten. Nachfolgend berichtet Sabine Finster von der Supervision in einem Frauenhaus. Sie zeigt, dass in diesem betont antihierarchischen Kontext durch die zumeist bestehende Traumatisierung der Frauen eine besondere Sorgfalt im Umgang mit Übertragungsphänomenen notwendig ist. Neben psychotraumatologischen Kompetenzen benötigen Supervisorinnen hier auch ein besonderes Verständnis für unterschiedliche Kulturen, da viele Frauen, die Frauenhäuser aufsuchen, aus fremden Ländern kommen. Idealerweise entstammen die Supervisorinnen selbst einem Immigrationsmilieu. Bettina Gorißen berichtet von Supervision bei der Feuerwehr. Schon bei der Lektüre des Beitrages wird deutlich, dass die Aufgaben der Feuerwehr den üblichen Erfahrungsbereich von Beratern bei weitem übersteigen. Die Autorin zeigt auch,

Online publiziert: 15.04 .2014

(C) Springer Fachmedien Wiesbaden 2014

Dr. A. Schreyögg $(\bowtie)$

Breisgauer Str. 29, 14129 Berlin, Deutschland

E-Mail: info@schreyoegg.de 
dass ein Supervisor oder eine Supervisorin jedenfalls nicht zimperlich sein darf, wenn sie in diesem Milieu supervidieren möchte. Das heißt, sie ist zum einen mit extrem ,,harten Buschen" konfrontiert, deren Akzeptanz sie für ihre Arbeit braucht, zum anderen mit extremen Schädigungen von Menschen.

Im nächsten Beitrag von Jost Buschmeyer und Claas Triebel geht es um die Unterstützung ausländischer Existenzgründer und Gründerinnen mit akademischem Hintergrund. Anhand einer empirischen Untersuchung kommen die Autoren zu dem Ergebnis, dass für die Beratung dieser Zielgruppe nicht nur eine besondere kulturelle Sensibilisierung, sondern auch eine höhere Kompetenzorientierung nötig ist, als bisher für solche Beratungen angenommen. Martina Meyer-Schwickerath und Monika Maßßen untersuchten, welche Schlüsselkompetenzen Mentoren oder Coaches benötigen, um neue Studierende an Hochschulen unterstützen zu können. Die ermittelten Kompetenzen wurden nachfolgend von den Studierenden selbst in der Praxis angewandt. An Hand eines mittelständischen Pharmaunternehmens zeigen Heidrun Strikker, Franz-Peter Kesseler und Frank Strikker, wie sie durch eine konsequente Arbeit mit der Führung des Unternehmens dem gesamten System zu einer neuen Firmenkultur verhelfen konnten. Thomas Meuche und Nicole Hüttner präsentieren demgegenüber „Organisationsentwicklung im öffentlichen Dienst“. Sie zeigen, wie sie die Institutsgründung an einer Hochschule für Angewandte Wissenschaften auf diese Weise vorangebracht haben.

Im Diskurs greift Ferdinand Buer ein ethisches Thema auf: „Sinngebende Beratung für sinnvolle Arbeit", und er wird konkreter, indem er zehn Orientierungswerte präsentiert. In einem zweiten Diskursbeitrag erörtert Thomas Webers die notwendigen Veränderungen des Führungsverhaltens, die sich aus der Entwicklung der Social Media ergeben und die auch für Formen und Inhalte im Coaching relevant sind. 